\title{
ISO RESULTS ON STAR FORMATION AND EARLY STELLAR EVOLUTION
}

\author{
THIERRY MONTMERLE \\ Service d'Astrophysique \\ CEA/DSM/DAPNIA/SAp, Centre d'Etudes de Saclay \\ F-91191 Gif-sur-Yvette Cedex, France \\ AND \\ LENNART NORDH \\ Stockholm Observatory \\ S-13336 Saltsjöbaden, Sweden
}

\section{Introduction: YSOs vs. ISO}

With all its instruments, $I S O$ is sensitive to temperatures in the range $\sim 30-2000 \mathrm{~K}$. This range is particularly adapted to studies of dense interstellar matter (gas and dust) in cold clouds, and to material associated with very early stages of stellar evolution: circumstellar matter around lowmass stars (disks, envelopes), outflows generated by them and interactions with the surrounding medium.

In this brief review we shall concentrate on the early evolution of low-mass stars $\left(M_{\star} \lesssim 1 M_{\odot}\right)$, usually referred to as "Young Stellar Objects" (YSOs). Two broad categories of YSOs have been identified: protostars and T Tauri stars. Using their IR-mm spectral energy distributions (SED), these categories are in turn subdivided into "Classes" thought to reflect evolutionary stages (see, e.g., André \& Montmerle 1994, André 1997). They are interpreted as hybrid objects, featuring envelopes, disks, and outflows to varying degrees. Chronologically, the youngest protostars are "Class 0": their SEDs peak in the mm domain, and most of their mass is contained in a dense envelope at the start of gravitational collapse (age scale $\tau \sim 10^{4} \mathrm{yrs}$ ). They are followed by "Class I" objects, their SED peaking in the mid- to far-IR. These are evolved protostars, in the sense that the central star is now essentially assembled, and is surrounded by a low-mass envelope and a circumstellar accretion disk $\left(\tau \sim 10^{5} \mathrm{yrs}\right)$. The protostar stages are also characterized by powerful molecular outflows (e.g., Bontemps et al. 1996). Then the envelope finally disappears, and all that remains is the disk, detectable as an SED excess in the near-IR: this is the "Class II" stage, in which the star becomes optically visible as a "classical" T Tauri star (CTTS; $\tau \sim 10^{6}$ yrs). Eventually (by an as yet unidentified mechanism, perhaps planet formation), this disk itself disappears (or at least becomes optically thin), leading to the "Class III" stage, or to "weak-line" T Tauri stars (WTTS) in the optical with no IR excess over a blackbody photosphere ( $\left.\tau \sim 10^{7} \mathrm{yrs}\right)$.

Of particular interest for $I S O$ are the stages with warm circumstellar material, i.e. the Class I and Class II YSOs, which can be observed out to long IR wavelengths with a much better angular resolution $(\times 10)$ and much greater sensitivity $(\times 10-50)$ than IRAS. For instance, the use of the broad-band LW2 $(\sim 7 \mu \mathrm{m})$ and LW3 $(\sim 15 \mu \mathrm{m})$ filters with ISOCAM allow to detect faint, heavily embedded Class II/CTTS objects: a Class II YSO with $A_{V}=20$ can be detected down to $L_{\star} \sim 0.01 L_{\odot}$ at the distance of the nearest star-forming regions $(d \sim 150 \mathrm{pc})$.

Because of space limitation, we have selected just a few highlights of results obtained by ISOCAM on nearby regions of star formation at large spatial scales, and by ISOCAM, PHOT and LWS on the environment of YSOs at small spatial scales. 


\section{Nearby regions of star formation: examples}

The $\rho$ Oph cloud $(d \sim 160 \mathrm{pc}$ ) is one of the closest and best studied regions of low-mass star formation. As part of the ISO Core Program on nearby star-forming regions, a wide $\left(\sim 0.75^{\circ} \times 0.75^{\circ}\right)$ mosaic of LW2/LW3 images has been obtained with ISOCAM (for details, see Abergel et al. 1996; Nordh et al. 1996, 1997). The combined 2-color image reveals a wealth of IR sources (Bontemps et al. 1997). As shown in Fig. 1, the colour index LW3/LW2 separates the sources into two groupings, one with mid-infrared excess ("red" objects, $\alpha>-1.5$ ) and one with normal photospheric colours ("blue" objects, $\alpha<-1.5$ ). The red objects can be safely identified with YSOs of Class I and II: with 73 new sources, the ISOCAM observations have more than doubled the number of known sources of this kind. The blue group represents a mixture of Class III YSOs and background objects (see Fig.1). It is likely that some of the blue ISOCAM objects, not earlier classified as YSOs, may also be young stars, but in order to clarify this point additional observations are needed. The same statement is true for the large number of objects detected only in LW2 (Olofsson et al. 1997).

The results obtained for $\rho$ Oph are similar to those obtained for the Cha I cloud (Nordh et al. 1997, Olofsson et al. 1997, Persi et al. 1997). For Cha I, Olofsson et al. (1997) have made a first attempt at deriving a luminosity function extending to very low luminosities. Using near infrared photometery to make 20 new YSO identifications from the group of blue ISOCAM sources and sources detected only in LW2, and by correlating IRAS luminosities when available (Prusti et al. 1992 ) with the LW2 fluxes, Olofsson et al. derived a surprisingly flat luminosity function for 65 sources, which extends down to $L_{b o l}=0.003 L_{\odot}$. Assuming a common age of $\sim 3 \times 10^{6}$ yrs for all the sources, they deduced the IMF using the pre-main sequence evolutionary models of D'Antona $\&$ Mazzitelli (1994). This IMF extends down to $\sim 0.02 M_{\odot}$, i.e. well below the brown dwarf limit $\left(0.08 M_{\odot}\right)$ : at face value, there are $\sim 20$ brown dwarfs in the sample. We caution, however, that this result is still preliminary and also rests on one particular choice of PMS models.

\section{The environment of YSOs}

$T$ Tauri disks. The SED of CTTS are usually modeled in terms of a combination of starlight (nearIR), and disk emission (mid- to far-IR). The disk may be heated either by the accretion process or by absorption of starlight (e.g., case of a "flaring" disk). Meyer et al. (1997) have analyzed PHOT data of CT Cha. The numerous new continuum spectral points constrain much better the SED and reveal a silicate emission feature around $10 \mu \mathrm{m}$, showing that in this star there may be indications of a residual envelope, corresponding to the very last phase of transition between a protostar and a T Tauri star.

Molecular outflows. An elongated outflow in the L1157 cloud, emanating from a deeply embedded Class 0 source, has been observed in ISOCAM/CVF mode by Cabrit et al. (1997). Fig. 2 shows a CVF image at $6.9 \mu \mathrm{m}$, i.e., the $0 \rightarrow 0 \mathrm{~S}(5)$ pure rotational line of $\mathrm{H}_{2}$. The ISOCAM image is strikingly similar to the $\mathrm{SiO}(2 \rightarrow 1)$ contours mapped in the mm radio range, which supports the interpretion of the IR emission in terms of a mild shock $\left(v_{s h} \sim 10 \mathrm{~km} \mathrm{~s}^{-1}\right)$ between the outflow and the surrounding cloud medium. An LWS spectrum of HH-54B taken by Liseau at al. (1996) also shows spectral evidences for a mild shock, in particular highly excited states of CO (up to $19 \rightarrow 18$ ), and of $\mathrm{H}_{2} \mathrm{O}$ (up to $3_{13} \rightarrow 2_{02}$ ). Similar mild shocks have been inferred from SWS observations of rotational lines of $\mathrm{H}_{2}$ in Cep A (Wright et al. 1996). These previously unrecognized shocks carry a significant amount of energy: in $\mathrm{HH}-54 \mathrm{~B}$, the total luminosity in the $\mathrm{H}_{2}$ line alone is estimated at $\sim 0.3 L_{\odot}$, i.e., about $10 \%$ of the outflow luminosity, itself a sizable fraction of the luminosity of the exciting source $\left(L_{b o l} \sim 10 L_{\odot}\right)$.

\section{Concluding remarks}

With its imaging and spectroscopic capabilities, ISO allows major advances in our knowledge of the cold, dense ISM, prior to star formation, and of early stages of stellar evolution. In particular, ISOCAM demonstrates enhanced capabilities for detection and characterization of low-mass YSOs and of their environment. PHOT allows much improved constraints on modelling of cold circumstellar material, while the CVF and the spectrometers (LWS and SWS) have obtained spectacular results on molecular gas shock chemistry, totally inaccessible from the ground. Only "the tip of the iceberg" has been analyzed, and much more is to come. 

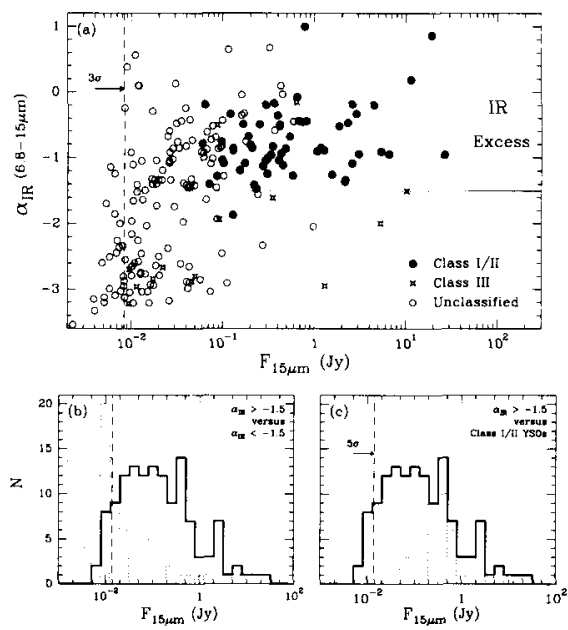

Figure 1. (a) Spectral index $\alpha_{I R}$ between the LW2 and LW3 ISOCAM filters, as a function of the LW3 flux $\left(\log F_{15}\right)$; (b) histogram for sources with $\alpha_{I R}<-1.5$ (continuous lines) vs. $\alpha_{I R}>-1.5$ (dotted lines); (c) same, vs. histogram of pre-ISO YSOs (dotted lines). (From Bontemps et al. 1997.)

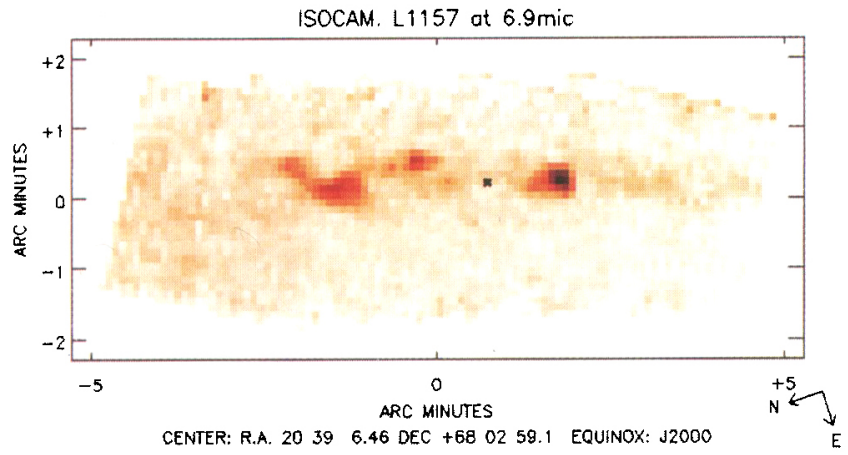

Figure 2. ISOCAM CVF image at $6.9 \mu \mathrm{m}$ (pure rotational line of $\mathrm{H}_{2}$ ), of the outflow excited by a Class 0 protostar (cross) in the L1157 cloud. This object is detected in particular in the LW9 filter, which does not contain any $\mathrm{H}_{2}$ line, suggesting dust continuum emission. (From Cabrit et al. 1997.)

\section{References}

Abergel, A. et al. 1996, A\&A, 315, L329

André, P. 1997, in Herbig-Haro Flows and the Birth of Low-Mass Stars, eds. B. Reipurth \& C. Bertout (Kluwer: Dordrecht), p. 483

André, P. \& Montmerle, T. 1994, ApJ, 420, 837

Barsony, M. et al. 1997, ApJS, 112, 109

Bontemps, S. et al. 1996, A\&A, 311, 858

Bontemps, S. et al. 1997, Proc. Star Formation with ISO (Lisbon), in press

Cabrit, S. et al. 1997, Proc. Star Formation with ISO (Lisbon), in press

D'Antona, F., \& Mazzitelli, I. 1994, ApJS, 90, 467

Liseau, R. et al. 1996, A\&A, 315, L181

Meyer, M., Beckwith, S., \& Natta, A. 1997, in Low-mass star formation (IAU Symp. 182), Poster proceedings, eds. F. Malbet \& A. Castets, p.224

Nordh, L. et al. 1996, A\&A, 315, L185

Nordh, L. et al. 1997, Proc. Star Formation with ISO (Lisbon), in press

Olofsson, G. et al. 1997, Proc. Brown Dwarfs $\mathscr{E}$ Extrasolar Planets (Tenerife), in press

Persi, P. et al. 1997, Proc. Star Formation with ISO (Lisbon), in press

Wright, C.M., et al. 1996, A\&A, 315, L301 